EPJ Web of Conferences 41, 02017 (2013)

DOI: $10.1051 /$ epjconf/20134102017

(C) Owned by the authors, published by EDP Sciences, 2013

\title{
Probing the longitudinal momentum spread of the electron wave packet at the tunnel exit
}

\author{
Claudio Cirelli ${ }^{1}$, Adrian N. Pfeiffer ${ }^{1}$, Alexandra S. Landsman ${ }^{1}$, Mathias Smolarski ${ }^{1}$, Darko \\ Dimitrovski ${ }^{2}$, Lars B. Madsen ${ }^{2}$, and Ursula Keller ${ }^{1}$ \\ ${ }^{1}$ Physics Department, ETH Zurich, CH-8093 Zurich, Switzerland \\ ${ }^{2}$ Lundbeck Foundation Theoretical Center for Quantum System Research, Department of Physics \\ and Astronomy, Aarhus University, 8000, Aarhus C, Denmark
}

\begin{abstract}
We present an ellipticity resolved study of momentum distribution arising from strong-field ionization of helium. The influence of the ion potential on the departing electron is considered within a semi-classical model consisting of an initial tunneling step and subsequent classical propagation. We find that the momentum distribution can be explained by including the longitudinal momentum spread of the electron at the exit from the tunnel. Our combined experimental and theoretical study provides an estimate of this momentum spread.
\end{abstract}

In strong-field physics and attoscience, it is often assumed that tunnel ionization is the first step that initiates the subsequent dynamics [1]. Therefore, the understanding of the electron-parent ion interaction in the presence of a femtosecond laser pulse is of fundamental importance to draw conclusions on various types of experiments. Providing a physical insight into this interaction, semiclassical models are indispensable for guiding ultrafast experiments and inventing new ultrafast measurement techniques such as tomography of molecular orbitals [2] and the attoclock [3] for example.

In the semiclassical model of strong-field ionization different steps explain the overall dynamics starting with the initial tunnel process followed by classical trajectories and potential rescattering or recombination. In a recent attoclock experiments [4] we obtained experimental information about the electron tunneling geometry (the natural coordinates of the tunneling current flow) and showed the importance of accurately accounting for the effective potential with the exact tunnel exit in semiclassical models. However, to date we did not address the momentum space distribution of the electron wavepacket at the tunnel exit. The momentum spread of the electronic wavepacket in the direction transverse to the field has been extensively studied both theoretically and experimentally [5]. Here we present experimental results, which can be explained with an initial longitudinal momentum spread of the electron at the tunnel exit.

The momentum spread of the electronic wavepacket at the tunnel exit point in the longitudinal direction has recently raised substantial interest. Tunneling theories impose the longitudinal spread at the tunnel exit to be zero [6]. However, a zero initial longitudinal momentum spread has failed to explain our experimental results. We measured the ion momentum distributions arising from strongfield ionization of helium in the tunneling regime, recorded over the full range of ellipticity. By comparing the experimental momentum distributions with the classical trajectory Monte Carlo (CTMC) simulations based on the Tunnel Ionization in Parabolic coordinates with Induced dipole

This is an Open Access article distributed under the terms of the Creative Commons Attribution License 2.0, which permits unrestricted use, distribution, and reproduction in any medium, provided the original work is properly cited. 
and Stark shift (TIPIS) model [4], we find that the momentum distributions strongly depend on the longitudinal electron momentum at the exit of the tunnel. In this sense, our experiment directly probes the longitudinal momentum spread of the electron at the tunnel exit point.

The experimental setup is explained elsewhere [4] therefore the description here is kept brief. Short laser pulses with a duration of $33 \mathrm{fs}$ (FWHM) at a central wavelength of $788 \mathrm{~nm}$ are produced by a Titanium:Sapphire based laser system and directed towards the helium target prepared as a supersonic jet in a COLd Target Recoil Ion Momentum Spectroscopy (COLTRIMS) setup [7]. We record the momentum distribution of helium ions for different values of ellipticity of the laser pulses, while keeping the intensity constant at a value of about $0.8 \mathrm{PW} / \mathrm{cm}^{2}$. In our experimental setup perfect circularly polarized pulses $(|\varepsilon|=1)$ cannot be generated. Due to the specification of the quarter-wave plate used, a maximum ellipticity of $\varepsilon= \pm 0.93$ was achieved.
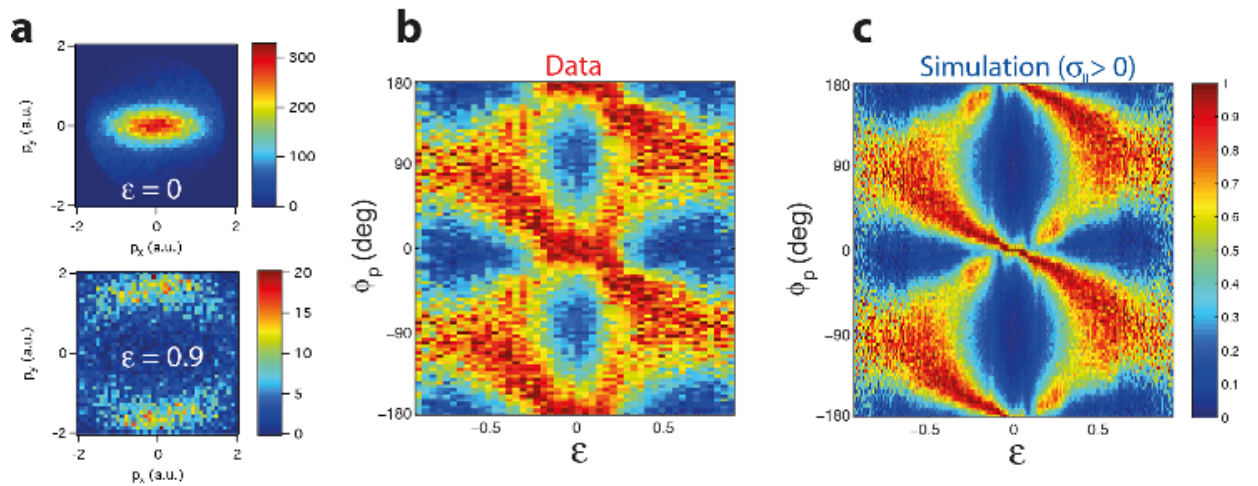

Fig. 1. a) Ion momentum distributions in the polarization plane arising from strong-field ionization for two selected ellipticities $(\varepsilon=0$ and $\varepsilon=0.9)$ : the shape of the distribution strongly depends on the laser polarization. The major polarization axis is the x-axis, the minor polarization axis is the y-axis. b) Measured ellipticitydependent angular distributions, where $\phi_{\mathrm{p}}=\arctan \left(\mathrm{p}_{\mathrm{y}} / \mathrm{p}_{\mathrm{x}}\right)$ is the radial component in polar coordinates of the ion momentum $\mathbf{p}=\left(\mathrm{p}_{\mathrm{x}}, \mathrm{p}_{\mathrm{y}}\right)$ shown in a). c) Angular distributions simulated with the CTMC simulations based on the TIPIS model, with an ellipticity dependent nonzero initial longitudinal momentum spread. The counts of the images in b) and c) are separately normalized for each fixed value of ellipticity in order to increase visibility.

The ion momentum distributions emerging after ionization differ qualitatively for linear $(\varepsilon=0)$ and circular polarization $(|\varepsilon|=1)$. For $\varepsilon=0$ the distribution in the polarization plane is close to Gaussian both along and perpendicular to the electric field direction; the radial component of the ion momentum $\phi_{\mathrm{p}}=\arctan \left(\mathrm{p}_{\mathrm{y}} / \mathrm{p}_{\mathrm{x}}\right)$ exhibits a maximum along the field direction $\left(\phi_{\mathrm{p}}{ }^{\max }=0,180\right.$ degrees, Fig 1b). In the case of close-to-circular polarization a torus forms around the center (Fig. 1a) and the maximum of $\phi_{\mathrm{p}}$ is located approximately in the direction of the minor axis (y-axis) of the polarization ellipse $\left(\phi_{\mathrm{p}}{ }^{\max }=90,270\right.$ degrees, Fig $\left.1 \mathrm{~b}\right)$.

For values of ellipticities in between, a cross-over takes place: the angular distribution has two distinct peaks for linear polarization and close to circular polarization, but has 4 peaks in the region around $\varepsilon= \pm 0.3$. The 4 peaks are clearly visible in Fig. $1 \mathrm{~b}$.

As stated above, in semiclassical models the initial electron momentum is assumed to be zero in a first approximation, but our results suggest that a more detailed description of the electronic wavepacket at the tunnel exit is necessary. Indeed it is possible to model the initial momentum distribution of the electron wavepacket at the tunnel exit point, providing different initial conditions for the semiclassical simulation.

The initial momentum distribution is usually discussed in terms of its components transversal and longitudinal to the electric field. In tunneling theory, a momentum distribution longitudinal to the field direction has been derived for elliptical polarization only after propagation in the laser field and presents a standard deviation $\sigma_{\|}^{\text {final }}$ as given in [8], which is a generalization for any ellipticity of the 
standard formula presented in [6] for linearly polarized fields. However, the initial longitudinal spread $\sigma_{\|}$is often assumed to be zero.

Performing semiclassical calculations with this assumption does not reproduce the experimental data (not shown here). We therefore assume that the initial momentum distribution longitudinal to the polarization is Gaussian with a standard deviation $\sigma_{\|}$and try different values for $\sigma_{\|}$in the semiclassical simulation. For all ellipticities we find a value for $\sigma_{\|}$greater than zero that yields a good agreement between data and simulation (Fig. 1 and Fig. 2).

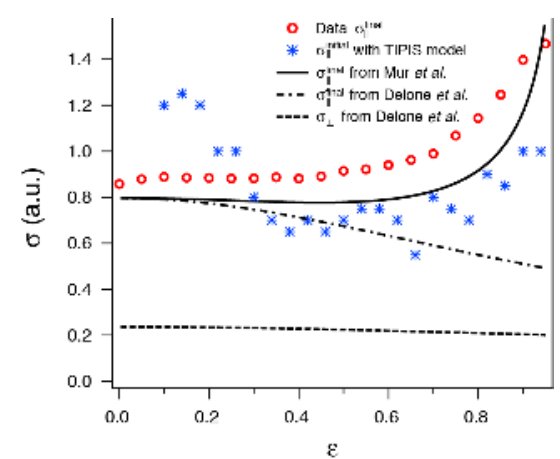

Fig. 2: The blue stars display the value for initial $\sigma_{\|}$chosen in the semiclassical calculations, that yields the least square-error with respect to the data shown in Fig. 1b. The red circles are the experimental data for the final momentum spread in the $\mathrm{x}$ direction, showing a good agreement with the theoretical curve predicted in [8], depicted as a solid line. For comparison the values for the final momentum spread in the perpendicular (dashed line) and parallel (dot-dashed line) direction introduced in [6] are also shown.

It is interesting to note that for $|\varepsilon|<0.3$, where the formation of the 4 peaks is the dominant feature in the momentum distribution, the final momentum spread is smaller than the initial spread at the tunnel exit. A possible reason for this is the presence of multiple returns of the liberated electron to the proximity of the ion core. For values of ellipticity larger than 0.3 the trend is reversed: the optimal momentum spread at the exit of the tunnel is smaller than the one measured at the detector, the latter being in fairly good agreement (within $10 \%$ error) with theoretical predictions.

In conclusion the good agreement between the experimental data and the numerical semiclassical model provides an evidence of the existence of the momentum spread of the electronic wave packet in the longitudinal direction at the tunnel exit point and enables the estimation of its value as a function of ellipticity.

1. F. Krausz, and M. Ivanov, Rev. Mod. Phys 81, 163 (2009)

2. J. Itatani, J. Levesque, D. Zeidler, H. Niikura, H. Pepin, J. C. Kieffer, P. B. Corkum, and D. M. Villeneuve, Nature 432, 867 (2004)

3. P. Eckle, A. N. Pfeiffer, C. Cirelli, A. Staudte, R. Dörner, H. G. Muller, M. Büttiker, and U. Keller, Science 322, 1525 (2008)

4. A. N. Pfeiffer, C. Cirelli, M. Smolarski, D. Dimitrovski, M. Abu-samha, L. B. Madsen, and U. Keller, Nature Phys. 8, 76 (2012)

5. L. Arissian, C. Smeenk, F. Turner, C. Trallero, A. V. Sokolov, D. M. Villeneuve, A. Staudte, and P. B. Corkum, Phys. Rev. Lett. 105, 133002 (2010)

6. N. B. Delone, and V. P. Krainov, J. Opt. Soc. Am. B 8, 1207 (1991)

7. R. Dörner, V. Merger, O. Jagutzki, L. Spielberger, J. Ullrich, R. Moshammer, and H. SchmidtBöcking, Phys. Rep. 330, 95 (2000)

8. V. D. Mur, S. V. Popruzhenko, and V. S. Popov, J. Exp. Theor. Phys. 92, 777 (2001) 